\title{
Embedding Systems Engineering Practices into Systems Engineering Classes
}

\section{Dr. S. Gary Teng, University of North Carolina, Charlotte}

Dr. S. Gary Teng is Professor of Systems Engineering \& Engineering Management and Director of Center for Lean Logistics and Engineered Systems at the University of North Carolina at Charlotte. He holds a P.E. license in the State of Wisconsin and is an ASQ-certified Quality Engineer and Reliability Engineer. His research interests are in engineering system design, analysis and management, supply chain management, lean systems, and risk management. Dr. Teng received the Bernard R. Sarchet Award in the Engineering Management Division of ASEE in June 2012 for his accomplishment in engineering management education. Between 2009 and 2012, he served on North Carolina's Governor's Logistics Task Force. The Task Force's mission is to enhance North Carolina's ability to move goods, people, and information efficiently and cost effectively. 


\title{
Embedding Systems Engineering Practices into Systems Engineering Classes
}

\begin{abstract}
The objective of this paper is to discuss the effect of enforcing Systems Engineering (SE) principles and practices into course work that can enhance students' understanding of the issues in systems integration and project management. In the process of educating SE students, professors need to ensure that students understand the importance of obtaining such skills as effective communications, good leadership, excellent project management, strong collaboration skills, high ability to adapt to multi-culture environments, and sufficient risk management ability. In addition, SE students also need to adopt two fundamental Industrial Engineering/SE principles, simplification and standardization, into their work. The issue is how we embed the requirements of these skills and SE principles into course work to educate SE students.

This paper intends to discuss the ways to teach students with SE principles and practices through a course called Systems Engineering Concepts. The discussion also points out the ways to enhance the education and training being offered to the SE students in order to obtain an adequate level of SE skills.
\end{abstract}

\section{Introduction}

SE practices are getting more critical in current global business environment since engineering and business projects and works require the collaboration of multiple partners in global locations. Engineering graduates who work on engineering related projects need adequate training and education to handle system related complex issues while performing their work.

The main function of SE is the design of an effective system and the integration of all system elements into a system that can achieve system objectives efficiently and provide optimal values to customers at the same time. Systems engineers deal with issues of all elements of a system, and they need to ensure that all participants understand the ultimate goal of the system while working on their subsequent tasks. Therefore, SE graduates have to obtain sufficient backgrounds and abilities to handle these system related issues.

This paper discusses the approach to teach SE principles and practices to students through a Systems Engineering Concepts course. The approach includes the use of a group project, project reports and presentations, one-page reports, the specification for the required file name format and project reports, and case studies that include Dabbawalla system, Boeing 787 Dreamliner production, and X-plane design competition. The discussions are ended with how to enhance the education and training being offered to the SE students in order to obtain an adequate level of SE skills.

\section{Research Objective}

The objective of this paper is to convene the effect of enforcing Systems Engineering (SE) principles and practices into the course work that can enhance students' understanding of the 
issues in systems integration and project management. The critical issue emphasized in this work is how we embed the requirements of these skills and SE principles into course work to educate SE students.

\section{The Approach}

To educate future systems engineers, professors need to ensure SE students to obtain sufficient abilities of having effective communications, good leadership, excellent project management, strong collaboration skills, better ability to adapt to multi-culture environments, and solid risk management.

The work described in this paper involves the offering of a sophomore SE course in a B.S. in SE curriculum, a course called Systems Engineering Concepts. This course offers SE students the knowledge in systems engineering process, systems design, and systems integration concepts. It is essential to let students understand the importance of implementing systems engineering process and the efficient integration of all aspects of system operations.

It is vital to let SE students realize that they need to adopt two fundamental Industrial Engineering/SE principles, simplification and standardization, into their work to make system operations successful to achieve overall system objectives. One of the issues for engineering educators is how we embed the requirements of the required SE skills and SE principles into class work to educate SE students.

In addition to regular SE lecture contents, examinations, and quizzes, the approach used in this Systems Engineering Concepts course includes the use of a group project, project reports with presentations, one page reports, the specification for file name format \& submissions, and case studies that include Dabbawalla system, Boeing 787 Dreamliner production, and X-plane design competition.

\section{Case Studies}

In this course, several case studies are discussed in class lectures, and used in assignments and in a term project. The purpose of having multiple case studies is to offer SE students an effective way to understand global business and engineering operations, systems and engineering issues, and how market competitions influence systems design through real industry cases. Cases being used in this course are listed as follows:

1. Boeing 787 Dreamliner production delay case ${ }^{1,2}$

2. X-Plane (X-35/X-32) fighter design competition case ${ }^{3}$

3. Mumbai India Dabbawalla system case ${ }^{4}$

4. Apple Store case

5. iPhone/iPad value distribution case

6. China EMS manufacturing facility case

7. US-China joint manufacturing facility case

8. Axle assembly line improvement case 
Using these cases to discuss systems engineering process and systems thinking as well as their implementations, the instructor provides some discussion points that reflect the issues of systems thinking and the achievement of systems objectives. For example, one discussion is on the initial implementation of lean approach to outsource various processes to multiple suppliers located at different countries for Boeing 787 Dreamliner's design and production activities. Some of the issues discussed include how the objectives of Boeing's outsourcing would have achieved company's business objectives, how suppliers should have worked on to achieve Boeing's objectives, what kind of risks were involved in Boeing's approach and how to manage these risks, how to deal with multi-culture issues in the Boeing 787 supplier network, and what Boeing CEO and Dreamliner project leaders should have done at the beginning of the project to make the project successful in the first time.

Some of the case studies offer the opportunity to deliberate the issues occurred in multi-culture business/engineering environments, such as the Dreamliner supplier network issues, the Dabbawalla system's successful operations, the manufacturing environment features associated with the China facility case along with the US-China joint facility case, and some practices in the Axle line improvement case. Multi-culture influence in systems design and systems operations is one of main points for the discussion. The emphases of these discussions are on how to embed multi-culture considerations into systems design and implementation, how to obtain adequate capability to deal with potential multi-culture issues in system operations, why some operations are successful under certain types of environments, and how to deal with potential risks caused by multi-culture environments.

The discussions also point out the importance of including multi-culture considerations and risk management strategies while defining system objectives and developing systems approach for the work. Students are also being asked to think about these issues from CEO's or senior management's perspectives and determine what they would have done differently to enhance these systems. Another emphasis of the discussion is on the flow of value in a system or in a supply chain and how to connect individual values defined by system participants to the ultimate system value and customer needs. Apple store case shows the example of creating a new value for Apple Inc. whose Apple stores generate the highest sales per square foot of floor space performance even though the initial purpose of having the stores was mainly for branding and marketing.

\section{Group Term Project}

SE students in the class need to complete a group term project at the end of the semester. The project assignment is based on the Boeing 787 Dreamliner production case. Student teams are required to investigate the Dreamliner's production delay issues and work on an area for systems improvement to make the 787 supply chain operations more efficient. All students get a project assignment sheet and a term project manual for the project work.

This term project manual offers the standards for the requested report formats and presentation requirements. The project assignment sheet clearly specifies project assignment objectives, project requirements, and project assignment milestone timeline. The project assignment includes four major milestones shown below: 
1. Project title and team member list submission

2. Proposal and presentation file submissions and proposal presentation

3. Progress report and presentation file submissions and progress report presentation

4. Final report along with presentation file submissions, and final report presentation

The first task for the students is to recruit classmates to establish a 4-person to 5-person team, investigate the case, and determine what area of the operations the grouped team wants to work on. Students have been constantly reminded the importance of task assignment and responsibility sharing to the success of the project results in the first few weeks of the class. The importance of leadership and collaborations is emphasized during their project work. In addition to the technical part of project tasks, students also need to work on their skills for leadership, project management, communications, and collaborations.

The project requirement calls for all students to present at least once or twice among three required team presentations. Students evaluate other team's presentations, and perform peer evaluations on their own teammates' contribution to their project. Peer reviews done at proposal and progress report milestones include providing each team member's percentage of contribution to the project work to date. Peer review at the final report milestone includes a seven category comprehensive evaluation of all project team members. Students understand that both good leadership and effective collaborations are critical for the success of their project works.

Team presentation evaluations include six to seven categories of each team's presentation. Students do not evaluate their own team's presentation performance. The purpose is to build up an SE student's ability to digest presentation contents quickly and make a decision on the quality of the presentation contents and the presentation itself. With the experience of evaluating presentations, SE students are aware of the significance of satisfactory technical contents, clear project objectives, good visual aid design, smooth transitions between team member's talks, effective conclusion, and accurate time control to a successful presentation.

At the end, most students not only understand their own team's project work, but also recognize the works done by other teams. They are able to ask in-depth questions, and provide constructive comments and suggestions. At the beginning of the project, the instructor always reminds the students to treat their project work as running a business with a vision. This request guides student teams to approach their project work from a higher system/company level perspective. It helps them look at the project issues more thoroughly and guide them to consider more system level elements in their Dreamliner project.

\section{Assignments}

Class assignments given to the students are all related to different cases and require students to search for related articles and information, analyze the information, build their own thoughts on the issue(s), and form an opinion on the issue(s). Since SE jobs require systems engineers to provide effective and get-to-the-point written communications, all class assignments are with a one-page report requirement which forces students to quickly pinpoint to the issues and their thoughts in the report and refine their writings to be more effective. 
The cases offered in the assignments are listed below:

1. Evaluate Boeing's current development of 787 and the new upgraded version of 737 (737MAX). From systems perspective, explore how Boeing competes with Airbus for similar markets and with Brazil's Embraer for smaller jet markets.

2. Discuss the importance of systems engineering process in the competition between Boeing and Lockheed Martin to pursue the X-plane contract from DoD.

3. Discuss how and why a design team needs to incorporate Poka-Yoke concept in systems design. For the application of Poka-Yoke concept in railroad intersection safety improvement, show if there is an even better way than the approaches in the provided figure to improve the intersection's safety assuming that (a) cost is not a main concern and (b) cost is a main concern.

These assignments are for the students to understand the issues from a system/company perspective and lead them to consider the influence caused by business and market competition, the complexity related system design problems, and the value related viewpoints for systems design and implementation.

\section{Video Clips Used}

In this course, students have the opportunities to view several video clips associated with the case studies. These video clips are available online and very useful for students to gain better understanding of the cases and their system issues. The available video clip categories are as follows:

1. Dabbawalla video clips (for a Mumbai lunch box delivery system)

2. Battle of the X-Planes video (for X-Planes design competition)

3. Boeing 787 video clips (for Boeing 787 Dreamliner design and production)

\section{Enforcement of Specifications}

In this course, students are required to follow all specified guidelines that include required submission file name formats, submission deadlines, assignment page limit and format, and project report requirements and formats. The importance of following specifications has been brought to students' attention repeatedly in the classroom since it is a systems engineer's job to ensure that all participants will follow the required specifications while doing their work.

It is extremely critical for students to understand that they need to follow the required specifications while doing their work and they should take it seriously. In addition to point reductions for not following the specifications, the instructor also shows them the result of inconsistency, and discusses the potential consequence caused by the violations of specifications. Figure 1 shows the report file name lists for the project reports. The lists are for nine project teams' project reports.

From this figure, it is obvious to see the great inconsistency occurred in proposal submission. The required file name format for proposal submission is "Team X-SEGR2101 proposal.doc(x)". 
